\title{
Alignment of nematic liquid crystals on an electrically poled photopolymer film
}

\author{
M. W. Kim a) and A. Rastegar \\ Research Institute for Materials, University of Nijmegen, 6525 ED-Nijmegen, Netherlands \\ I. Drevensek Olenik \\ Faculty of Mathematics and Physics, University of Ljubljana, Jadranska 19, SI 1000 Ljubljana, Slovenia \\ M. W. Kim ${ }^{\text {a) }}$ \\ Department of Physics, Korea Advanced Institute of Science and Technology, Daejon, Korea \\ Th. Rasing ${ }^{\text {b) }}$ \\ Research Institute for Materials, University of Nijmegen, 6525 ED-Nijmegen, Netherlands
}

(Received 9 October 2000; accepted for publication 10 May 2001)

\begin{abstract}
We have observed liquid crystal alignment on an electrically poled polyvinylcinnamate film in which a polar symmetry was introduced by an in-plane electric field and stabilized by unpolarized ultraviolet light. The azimuthal anchoring energy and pretilt angle was measured in a twisted nematic or planar cell and is explained via the molecular structure of the unreacted cinnamates and of the photoproducts $\beta$-truxinates. Cinnamates mainly contribute via a strong flow alignment, while $\beta$-truxinate produces the anisotropic properties of the film such as its polar anisotropy, azimuthal anchoring, and pretilt angle. (C) 2001 American Institute of Physics. [DOI: 10.1063/1.1383580]
\end{abstract}

\section{INTRODUCTION}

One of the key issues of liquid crystal displays (LCDs) is the uniform alignment of the liquid crystal (LC) molecules on an aligning surface. In practice this can be achieved by using a thin polymer film that provides defect free and anisotropic anchoring for the LCs.

There are several well known methods ${ }^{1-3}$ to introduce a surface anisotropy onto a polymer film. Among them the rubbing by a velvet cloth is most popular due to its simplicity and the possibility of controllable anchoring energy and pretilt angle. However, rubbing easily introduces dust and charges that may lead to serious damage in the final LCD production. ${ }^{4}$ Therefore there has been a lot of research to develop rubbing free methods. ${ }^{5,6}$ As a promising alternative, Schadt et al. ${ }^{6}$ have introduced a photopolymer film exposed to linearly polarized ultraviolet light (LPUV). The LPUV method has the additional advantage that it can be applied to fine pixels, which is advantageous in obtaining wide view angles. Its disadvantages are unstable anchoring and no pretilt angle. Note that though on a molecular level there is a pretilt angle, this averages out to zero because of the two fold degeneracy of such systems (see Refs. 6 and 7).

We have recently demonstrated an electrically poled photopolymer (EPP) film ${ }^{8-10}$ as a rubbing free method to improve the anchoring properties of LPUV films. A fairly weak electric field in combination with a fixation using ultraviolet induced polymerization was shown to lead to an anisotropy of photopolymers that can be used for LC alignment. ${ }^{8,9}$

\footnotetext{
a) Also at: Department of Advanced Materials Engineering, Korea Advanced Institute of Science and Technology, Seoul, Korea.

${ }^{\text {b)} E l e c t r o n i c ~ m a i l: ~ t h e o r a s @ s c i . k u n . n l ~}$
}

In this article we report on the mechanisms of nematic liquid crystal alignment on such an EPP film. In particular the observation of the azimuthal anchoring energy in twisted nematic (TN) cells and the pretilt angle in planar cells are presented and their alignment mechanisms are explained via the molecular structure of the EPP film.

\section{EXPERIMENT}

The preparation of the EPP film has been discussed before $^{8-10}$ In short, thin films of polyvinylcinnamate (PVCN) were prepared by spin- or dip-coating a solution of $1 \mathrm{wt} \%$ PVCN (Aldrich) in chloroform on a glass substrate between two indium-tin-oxide (ITO) coated electrodes that were $3 \mathrm{~mm}$ apart. The thickness of the film, measured using a thickness profiler and ellipsometry, was about $400 \pm 100$ $\mathrm{nm}$. The coated films were dried for $1 \mathrm{~h}$ at $100^{\circ} \mathrm{C}$ in an oven to remove the solvent. The samples were poled at $55 \pm 1{ }^{\circ} \mathrm{C}$ by applying an electric field of $2 \mathrm{kV} / \mathrm{cm}$ for more than 30 min and exposed to unpolarized UV light for various times from 0 to $60 \mathrm{~min}$. The UV light source was a $150 \mathrm{~W}$ Xe-arc lamp and was placed $22 \mathrm{~cm}$ from the sample. Before cell preparation, the optical anisotropy of the EPP films was measured by a sensitive birefringence setup. ${ }^{8}$

To determine the azimuthal anchoring energy, TN cells were prepared using an EPP film and a rubbed polyimide (PI) film as a reference surface (5-Cyano biphenyl on the rubbed PI surface was assumed to have a strong anchoring energy). The cell thickness was adjusted to $5.6 \mu \mathrm{m}$ using polyethyleneterephthalate (PET) films and was determined by the interference method. ${ }^{11}$ The cells were filled with $5 \mathrm{CB}$ in the nematic or isotropic phase. In order to investigate the effect of the electric field on the alignment, the field in a TN cell was oriented parallel, antiparallel, or perpendicular to the 
TABLE I. Twist angle relative to the rubbing direction of PI in an EPP cell.

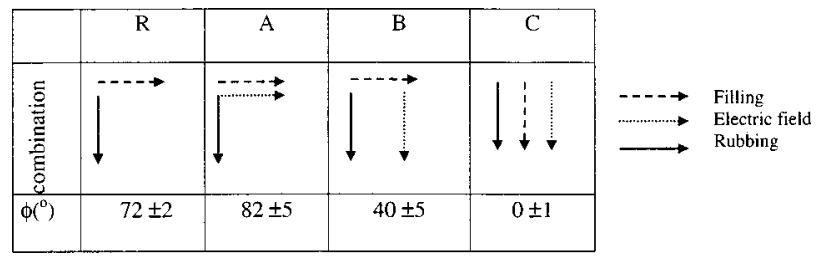

rubbing direction of the PI. To study the presence of a flow effect on EPP films, the cell was filled parallel, antiparallel, or perpendicular to the electric field. The twist angle in a TN cell was measured using the method given in Ref. 12 and used to calculate the azimuthal anchoring energy.

For pretilt angle measurements, planar cells were prepared using an EPP film and an ITO surface, that was rubbed five times by a homemade rubbing machine with a velvet cloth. The cell thickness was adjusted and measured using the same method as for the TN cells. The cells were filled parallel and antiparallel to the electric field with $5 \mathrm{CB}$ in the nematic phase. For the light source a He-Ne laser was used without focusing in order to measure a macroarea. The textures of the cells were observed under a microscope with crossed polarizers.

\section{RESULTS AND DISCUSSIONS}

\section{A. Azimuthal anchoring}

The twist angle in a cell with EPP and PI is very sensitive to the LC filling direction. Table I shows the obtained twist angles $\phi$ depending on the combination of three factors: the rubbing of PI, the electric field of the EPP, and the filling direction in the nematic phase of $5 \mathrm{CB}$.

The cells in Table I are all aligned with PI and an EPP film exposed to UV for 10 min except $R$ (with a PVCN film instead of an EPP one). The LC on PI has a strong anchoring energy $\left(10^{-4} \mathrm{~J} / \mathrm{m}^{2}\right) . R$ has a twist angle of $72 \pm 2^{\circ}$ due to the strong flow effect on PVCN. The flow direction in a cell with an EPP film is seen to determine the LC director on the film, while the electric field plays a secondary role in the alignment. Depending on the filling $A$ parallel to the electric field achieves a TN structure and $B$, perpendicular to the field is only half twisted by rotating the flow alignment to the field direction, while $C$, with the three factors parallel to each other shows a planar structure.

The above comparisons lead to the conclusion that the anchoring energy on an EPP film is determined by the flow and the electric field of the EPP film and its maximum is obtained by having these two factors in parallel.

The LC alignment texture of $A$ was studied with changing UV-exposure times under a crossed polarized microscope (see Fig. 1). A cell exposed for less than 30 min shows stripes of about a $100 \mu \mathrm{m}$ width and parallel to the filling direction. With increasing the exposure time to more than 40 min, the cells become homogeneous and defect free.

Figure 2 shows the azimuthal anchoring energy $W_{A}$ of $5 \mathrm{CB}$ in a TN cell as a function of unpolarized UV exposure time. $W_{A}$ is calculated from the twist angle of the TN cell by using $^{12}$

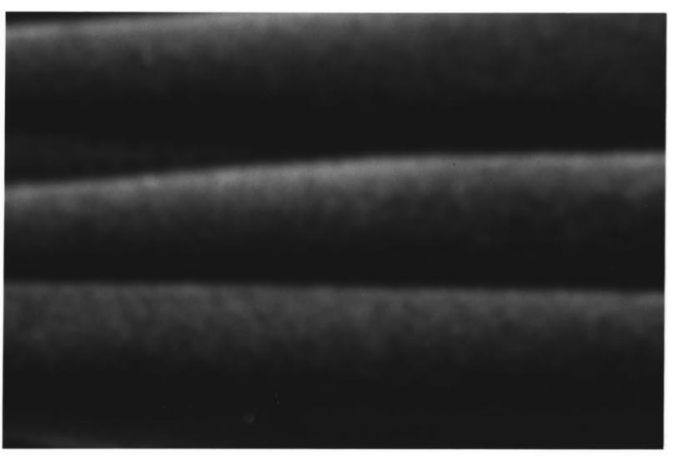

(a)

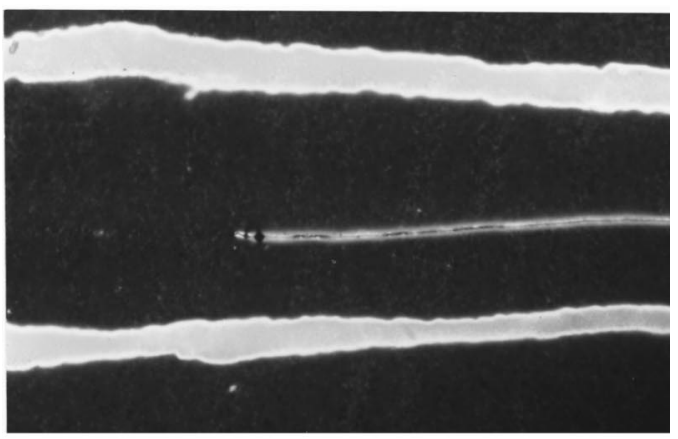

(b)

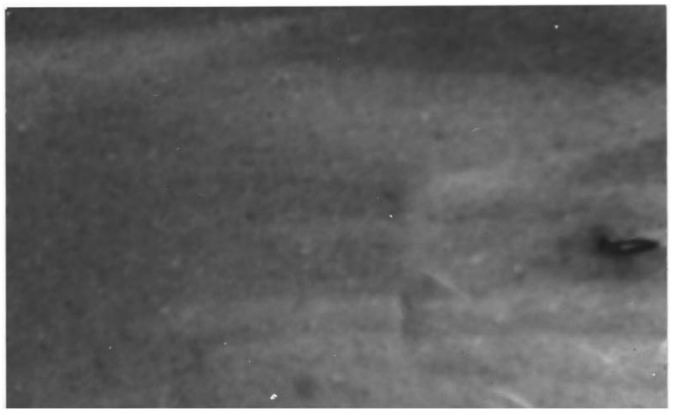

(c)

FIG. 1. Microphotograph of the liquid crystal texture in twisted nematic cells under crossed polarizers. The cells were aligned between an EPP film with various UV exposure times: (a) 10, (b) 20, and (c) $60 \mathrm{~min}$ and a Rubbed PI film and filled with 5CB parallel to the electric field of the EPP film in the nematic phase. The stripes for short unpolarized UV exposure times disappear with increasing time.

$$
W_{A}=\frac{2 K_{22} \phi}{L \sin 2 \phi},
$$

where $L$ is the cell gap and $K_{22}$ is the twist elastic constant of 5CB. The azimuthal anchoring energy shown in Fig. 2 increases first rapidly with UV exposure and after a maximum slowly reduces to a constant with extending UV exposure time.

In order to explain this observation, the azimuthal anchoring energy on an EPP film can be expressed via the van der Waals' interaction between the LCs and an EPP film and the anisotropy of an EPP film. ${ }^{13}$

$$
W_{A} \sim W_{s} \cdot S^{2}
$$

with $W_{s}=$ the van der Waals' interaction and $S=$ the LC order parameter. Starting with the van der Waals' interaction, the 


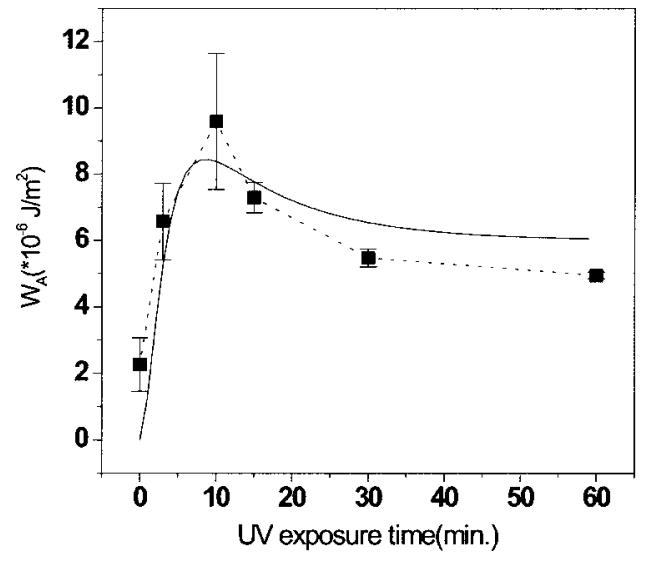

FIG. 2. Azimuthal anchoring energy of 5CB on an EPP film as a function of unpolarized UV exposure time (solid squares and dotted line). The solid line is a fit to Eq. (7) with $\alpha=0.008, \beta=0.28$, and $W_{0}=6 \times 10^{-6} \mathrm{~J} / \mathrm{m}^{2}$.

interaction energy $W_{s}$ on an EPP film can be described by a contribution from the cinnamates $\left(W_{c}\right)$ and $\beta$-truxinates $\left(W_{t}\right)$

$$
W_{s}=W_{c}+W_{t} \approx W_{c} .
$$

Due to the dominant interaction of the cinnamate groups with the LCs, ${ }^{14,15} W_{s}$ is approximated by $W_{c}$. Figure 3(a) shows the relative concentration of cinnamates $C_{c}$ as a function of UV exposure obtained from the UV spectra (Fig. 3, inset). The reduction of the interaction energy as a function of UV exposure time $\tau$ can be written as

$$
W_{s}=A(1+\exp (-\alpha \tau)),
$$

where $A$ is a constant defined as the interaction energy per area between the LCs and the PVCN film and $\alpha$ is the photoreactivity obtained as 0.08 via fitting $C_{c}$ as a function of $\tau$.

The increase of the optical anisotropy $\Delta_{s}$ [Fig. 3(b)] of a poled PVCN film during the UV exposure can be described with a scalar order parameter $S_{s} .{ }^{16}$ The anisotropic refractive index is equal to

$$
\Delta n_{s} \sim \rho^{1 / 2} S_{s},
$$

where $\rho$ is the density of the film that remains constant during the photoreaction. $\Delta_{s}$ shown in Fig. 3 can be fitted as a function of $\tau$ using

$$
\Delta_{s}=\int_{L} \Delta n_{s} \mathrm{~d} L=\Delta_{M}(1-\exp (-\beta \tau)),
$$

where $L$ is the thickness of the EPP film. $\Delta_{M}$ and $\beta$ are constants used to fit the curve in Fig. 3(b), yielding $\Delta_{M}$ $=0.003$ and $\beta=0.28$, respectively. $S_{s}$ can be replaced by the LC order parameter $S$ under the approximation that the LC order parameter is linearly proportional to the anisotropy of the film. Substituting into Eq. (2) this yields

$$
W_{A} \approx W_{0}(1+\exp (-\alpha \tau))(1-\exp (-\beta \tau))^{2},
$$

where $W_{0}$ is a constant. The azimuthal anchoring energy shown in Fig. 2 is fitted using Eq. (7), $\alpha$ and $\beta$ obtained above and $W_{0}=6 \times 10^{-6} \mathrm{~J} / \mathrm{m}^{2}$. The fit is presented as the solid line in Fig. 2 and explains the behavior of the azimuthal anchoring quite well. The maximum is an optimized azi-
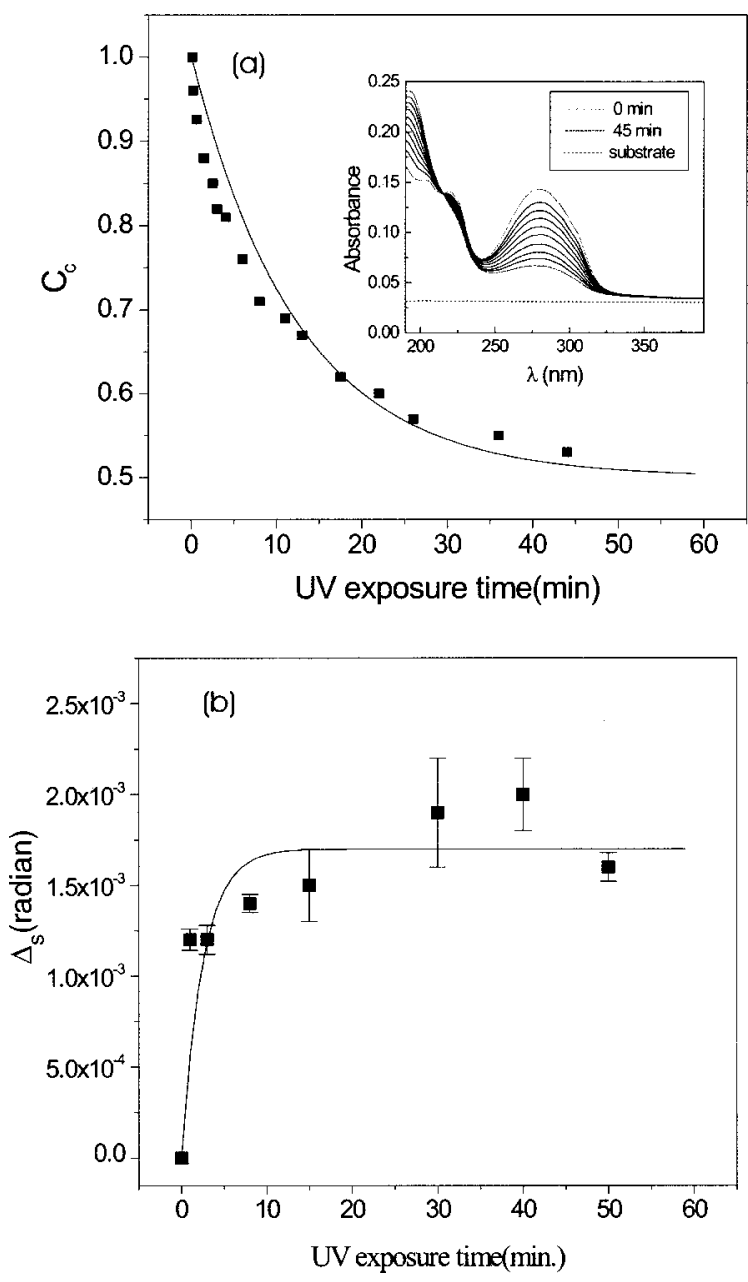

FIG. 3. inset Absorption spectra of a PVCN film as a function of unpolarized UV exposure time between 0 and $45 \mathrm{~min}$. (a) The relative concentration of cinnamate as a function of unpolarized UV exposure time, calculated using the absorbances at $250 \mathrm{~nm}$ and at $275 \mathrm{~nm}$ and the method given in Ref. 29. The solid line is a fit to Eq. (4) with $\alpha=0.028$. (b) the optical anisotropy of an EPP film as a function of unpolarized UV exposure time. The solid line is a fit to Eq. (6) with $\Delta_{M}=0.003$ and $\beta=0.28$.

muthal anchoring energy due to the decrease of the interaction energy and the increase of the order parameter during the photoreaction. One can estimate $S$ by using Eq. (2), taking $W_{A}=10^{-6} \mathrm{~J} / \mathrm{m}^{2}$ from Fig. 2 and $W_{s}=10^{-3} \mathrm{~J} / \mathrm{m}^{2}$ for the condition of a weak interaction between the two layers. ${ }^{17}$ This approximation gives for the order parameter of $5 \mathrm{CB}$ on EPP film $S \sim 10^{-2}$, which is 10 times smaller than $S$ of a rubbed $\mathrm{PI}^{18}$ but 10 times bigger than the $10^{-3}$ of an oblique evaporated $\mathrm{SiO}$ layer ${ }^{19}$ or LPUV PVCN. ${ }^{20}$

\section{B. Pretilt angle on an EPP film}

Figure 4 shows the relative optical transmittance as a function of the angle of incidence, which was measured by the crystal rotation method ${ }^{21}$ for two planar cells. The cells were filled with 5CB parallel (solid line) and antiparallel (dotted line) to the electric field. For the parallel configuration the symmetry angle of the curve is shifted to about $5^{\circ}$, while for the antiparallel one the angle is at $0^{\circ}$. The result indicates that only for the parallel configuration, the cell has a pretilt angle. 


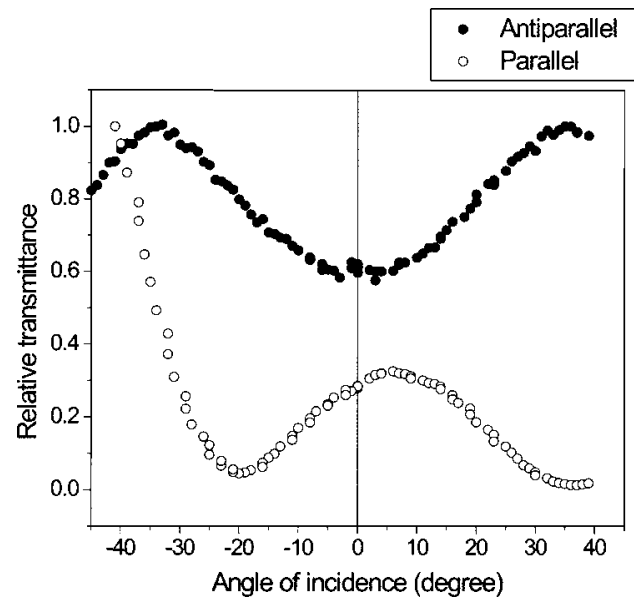

FIG. 4. Relative optical transmittance of planar cells as a function of the angle of incidence according to the crystal rotation method given in Ref. 2. The cells were aligned between a rubbed ITO film and an EPP film; closed circles, the electric field of an EPP film antiparallel to flow; open circles, electric field parallel to flow.

Figure 5 shows the pretilt angle of a planar cell as a function of unpolarized UV exposure time. All the cells were filled along the electric field direction. The pretilt angle $\theta_{s}$ on an EPP film is obtained by using ${ }^{7}$

$$
\bar{\theta}=\frac{\theta_{r}+\theta_{s}}{2},
$$

where $\bar{\theta}$ and $\theta_{r}$ are the angles measured from the crystal rotation method and the pretilt angle on a reference surface respectively (on rubbed ITO, the pretilt angle is $0.5^{\circ}$ and parallel to the rubbing).

According to Dyadyusha et al. ${ }^{22}$ the zero pretilt angle of an LPUV PVCN film is due to the nonpolar anisotropy axis of linearly polarized light. Therefore, even if the photoreacted surface provides an oblique orientation, this orientation should be twofold degenerate and their vector sum leads to a zero pretilt angle. Hence, in order to produce a pretilt angle, the anisotropy of an EPP film has to have a polar $C_{s}$ sym-

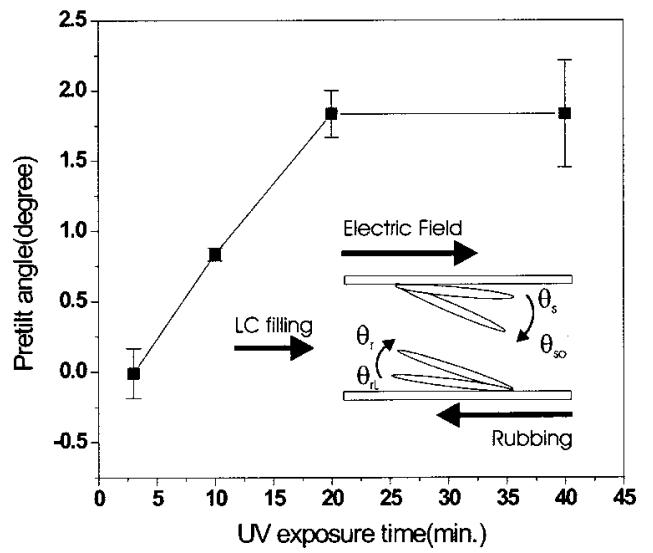

FIG. 5. Pretilt angle on the EPP film in a planar cell as a function of unpolarized UV exposure time. The planar cells were aligned between a rubbed ITO film and an EPP film exposed to unpolarized UV light for various times and filled parallel to the electric field of the EPP film with $5 \mathrm{CB}$ in the nematic phase. metry, which can be induced by an electric field. ${ }^{9}$ Therefore, the increase of the pretilt angle with UV exposure time as observed in Fig. 5 must be due to the dependence of the polar anisotropy on an EPP film on the UV exposure time. ${ }^{9}$

In order to find the relationship between the pretilt angle and the anisotropy of an EPP film, we write down the free energy of a planar cell as

$$
\begin{aligned}
f= & \frac{1}{2} \int_{0}^{L} K\left(\frac{d \theta}{d z}\right)^{2} d z+\frac{1}{2} W_{p} \sin ^{2}\left(\theta_{s}-\theta_{s o}\right) \\
& +\frac{1}{2} W_{r} \sin ^{2}\left(\theta_{r}-\theta_{r L}\right),
\end{aligned}
$$

where $K=1 / 3\left(2 K_{11}+K_{33}\right)$ and $W_{p}$ and $W_{r}$ are the polar anchoring energies of PVCN and ITO films, respectively. $\theta_{s}$ and $\theta_{r}$ are the pretilt angles on the $\operatorname{PVCN}($ at $z=0)$ and the ITO (at $z=L$ ) surface and $\theta_{s o}$ and $\theta_{r L}$ are the easy axis on the PVCN and ITO surface, respectively.

The boundary condition at the EPP film surface at $z$ $=0$ yields

$$
\frac{1}{2} W_{p} \sin 2\left(\theta_{s o}-\theta_{s}\right)-K \frac{d \theta}{d z}=0 .
$$

Having a weak anchoring $\left(\cong 10^{-6} \mathrm{~J} / \mathrm{m}^{2}\right)$ on the opposite ITO surface $\left(\theta_{r o}=0.5^{\circ}\right)$ and using the approximation $d \theta / d z$ $\approx \theta_{r}-\theta_{s} / L=\zeta$, Eq. (10) leads to

$$
\left(\theta_{s o}-\theta_{s}\right) \approx \frac{K \zeta}{W_{p}} .
$$

Equation (11) shows that the deviation of the LC anchoring from the easy axis, $\left(\theta_{s o}-\theta_{s}\right)$ of an EPP film is inversely proportional to the polar anchoring energy $W_{p}$.

According to Zhuang et $_{\text {al. }}{ }^{23}$ the polar anchoring energy is linearly proportional to the tensor order parameter $Q$ of a $\mathrm{LC}$ and defined by

$$
W_{p} \approx W_{s} Q .
$$

Comparing Eq. (12) with Eq. (11) shows that with increasing $W_{s} Q$, the pretilt angle will be closer to the easy axis of the EPP film, $\left(\theta_{s o}-\theta_{s}\right) \approx 0$. The inset of Fig. 5 shows the change of the director orientation at each surface of the cell. With increasing $\theta_{s}$, the $\theta_{r}$ follows. Therefore, the increase of the pretilt angle as a function of UV exposure time shown in Fig. 5 results from the increasing $W_{p}$ due to the increasing anisotropy of the EPP film.

Furthermore, $W_{s}$ consists of two terms: a decreasing $W_{c}$ and an increasing $W_{t}$ as a function of $\mathrm{UV}$ exposure time and Eq. (12) becomes

$$
W_{p} \approx W_{c} Q+W_{t} Q .
$$

Taking into account that the UV light is unpolarized, the cinnamates that are randomly decreasing in number on the film surface are hardly expected to contribute to the increase of the pretilt angle. Therefore, the first term of Eq. (13) is determined by flow alignment which acts against the pretilt angle when a cell is filled antiparallel to the electric field (see Fig. 4). The second term corresponds to the photoproducts from poled cinnamates and thus can be seen as the major factor that causes the increase of the pretilt angle. 
(a)

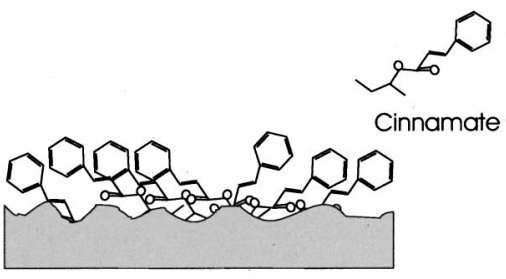

PVCN film Surface

(b)

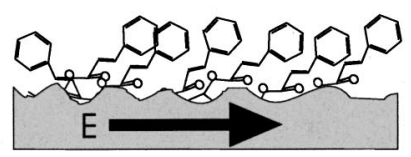

PVCN film after poling

(c)

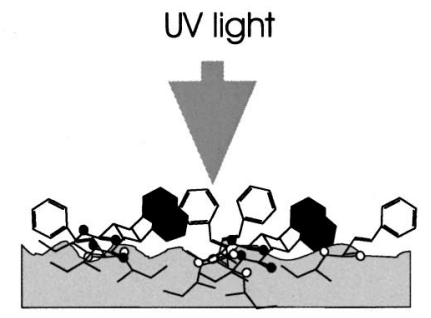

EPP film Surface after photoreaction

(d)

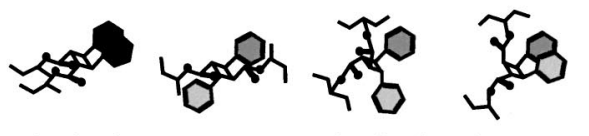

$\beta$-truxinate $\alpha$-truxillate $\delta$-truxinate neotruxinate

FIG. 6. (a) surface structure of a PVCN film. (b) poled PVCN film and (c) EPP film after unpolarized UV exposure. (d) four major photoproducts from the photoreaction of cinnamates.

\section{Mechanism of LC alignment based on the structure of an EPP film}

Surprisingly, we found the LC alignment on the weak anisotropy of an EPP film to be stable for more than one year. This stable alignment mechanism can be explained via the molecular configuration of the film surface.

A PVCN film is known to have microdomains ${ }^{6}$ and from our surface second harmonic generation investigations, we know that the cinnamates are tilted by $12^{\circ} \pm 5$ from the surface [see Fig. 6(a)]. ${ }^{24}$ The cinnamates will be aligned parallel to the electric field [see Fig. 6(b)] and photoreact with nearest neighbors. ${ }^{6}$ Taking into account the molecular configuration of two cinnamates participating in the photoreaction, the film surface can be expected to have $\beta$-truxinate as a major photoproduct in addition to some other isomers and left cinnamate groups after photostabilization. The resulting surface of the EPP film looks like the cartoon in Fig. 6(c).

From Fig. 3(a) it follows that approximately 50\% cinnamate remains after the photoreaction. ${ }^{25}$ These leftover cinnamates can explain the strong flow effect because of their strong interaction with liquid crystal molecules via the $\pi$-conjugation along the optic axis. ${ }^{25}$ This flow effect is also expected to be responsible for the high stability of the LC cells. ${ }^{26-28}$

Hence, an EPP film contains two important factors that play a role for aligning liquid crystals: the leftover cinnamates and the photoproducts. Considering the random distribution of cinnamates on the film surface, one can conclude that oriented $\beta$-truxinates contribute to the alignment and pretilt angle of the liquid crystals on an EPP film while the cinnamates mainly contribute via the flow memory to the stable alignment.

\section{CONCLUSIONS}

In conclusion, the polar anisotropy of an EPP film (achieved in a polyvinylcinnamate photopoymer film by electric field poling and photostabilization by unpolarized UV light) aligns LCs with high stability and pretilt angle.

The LC alignment properties are in general changed by the UV exposure time, while a strong anchoring is obtained by combining the flow and the anisotropy of the film. The observed optimum azimuthal anchoring energy of liquid crystals on an EPP film is due to the photoreaction and the changing anisotropy of an EPP film with increasing UV exposure time. During the photoreaction, the van der Waals interaction between the LC layer and the EPP film decreases due to the molecular structure change from the conjugated cinnamate to the nonconjugated truxinate, while the anisotropy of the film increases due to the stabilization of the poled cinnamates. Therefore, a maximum is obtained via the competition between these two factors as a function of UV exposure time.

A pretilt angle on an EPP film surface is achieved via filling a cell parallel to the electric field direction and this angle increases with UV exposure time. This is due to the increase of the polar anisotropy of the EPP film with UV exposure time.

There are two important molecular structures on an EPP film which determine the LC alignment: the photoproduct $\beta$-truxinate, contributing to the alignment of liquid crystals via the polar anisotropy and pretilt angle and the remaining cinnamates via the flow memory. From our observations, we may conclude that an EPP film is a promising method to align nematic liquid crystals with stable anchoring and pretilt angle.

\section{ACKNOWLEDGMENTS}

Part of this work was supported by the EU Network SILC and by the Stichting Technische Wetenschappen (STW).

${ }^{1}$ Ch. Ganwiller, Phys. Rev. Lett. 28, 1554 (1972).

${ }^{2}$ F. J. Kahn, G. N. Tayer, and H. Schonhorn, Proc. IEEE 61, 823 (1973).

${ }^{3}$ D. W. Berreman, Phys. Rev. Lett. 28, 1638 (1972).

${ }^{4}$ J. A. M. M. van Haaren, Nature (London) 381, 190 (1996).

${ }^{5}$ G. P. Bryan-Brown, E. L. Wood, and I. C. Sage, Letters to Nature 399, 338 (1999).

${ }^{6}$ M. Schadt, K. Schmitt, V. Kozinkov, and V. G. Chigrinov, Jpn. J. Appl. Phys., Part 1 31, 2155 (1992).

${ }^{7}$ A. Dyadyusha et al., Mol. Cryst. Liq. Cryst. 263, 399 (1995).

${ }^{8}$ M. W. Kim, A. Rastegar, I. Drevensek, P. de Witte, M. W. Kim, and Th. Rasing, Mol. Cryst. Liq. Cryst. 329, 441 (1999). 
${ }^{9}$ M. W. Kim, A. Rastegar, I. Drevensek, P. de Witte, R. Nolte, and Th. Rasing, Mol. Cryst. Liq. Cryst. Sci. Technol., Sect. A 352, 407 (2000).

${ }^{10}$ A. Rastegar, M. W. Kim, I. Drevensek, and Th. Rasing, Mol. Cryst. Liq. Cryst. Sci. Technol. Sect. A 351, 169 (2000).

${ }^{11}$ R. Sun, X. Haung, K. Ma, and H. Jing, SID Proc. IDRC, 225 (1994).

${ }^{12}$ E. L. Wood, G. W. Bradberry, P. S. Cann, and J. R. Sambles, Proc. SPIE 3318, 267 (1998).

${ }^{13}$ D. W. Berreman, Phys. Rev. Lett. 28, 1983 (1972).

${ }^{14}$ S. Perny, P. Barny, J. Belaire, T. Buffeteau, C. Sourisseau, I. S. Forget, and P. M. Lagarde, Liq. Cryst. 27, 329 (2000).

${ }^{15}$ H. G. Galabova, D. W. Allender, and J. Chen, Phys. Rev. E 55, 1627 (1997).

${ }^{16}$ A. P. Filippov, L. N. Andreeva, E. B. Barmatov, and V. P. Shibaev, Proc. SPIE 3318, 71 (1998).

${ }^{17}$ J. N. Israelachvili, Intermolecular Force and Surface Forces, 2nd ed. (Academic, New York, 1992).

${ }^{18}$ T. Isozaki, T. Fujkawa, H. Takazoe, A. Fukuda, T. Hagiwara, Y. Suzuki, and I. Kawamura, Jpn. J. Appl. Phys., Part 2 31, L1435 (1992).

${ }^{19}$ S. Faetti, M. Gatti, V. Palleschi, and T. J. Sluckin, Phys. Rev. Lett. 55, 681 (1985).

${ }^{20}$ A. Th. Ionescu, R. Barberi, M. Giocondo, M. Iovane, and A. L. AlexeIonescu, Phys. Rev. E 58, 1967 (1998).

${ }^{21}$ B. Jerome, Mol. Cryst. Liq. Cryst. 212, 21 (1992).

${ }^{22}$ D. Seo, Y. Limura, and S. Kobayashi, Appl. Phys. Lett. 61, 234 (1992).

${ }^{23}$ X. Zhuang, L. Marrucci, and Y. R. Shen, Phys. Rev. Lett. 73, 1513 (1994).

${ }^{24}$ I. Drevensek, M. W. Kim, A. Rastegar, and Th. Rasing, Phys. Rev. E 60, 3120 (1999)

${ }^{25}$ S. Murase, K. Kinoshita, K. Horie, and S. Morino, Macromolecules 30, 8088 (1997).

${ }^{26}$ J. Cheng and G. D. Boyd, Appl. Phys. Lett. 35, 444 (1979).

${ }^{27}$ B. Jerome and P. Pieransky, J. Phys. (France) 49, 1601 (1988).

${ }^{28}$ H. Yokoyama, S. Kobayashi, and H. Kamei, J. Appl. Phys. 56, 2645 (1984).

${ }^{29}$ L. Egerton, E. Pitts, and A. Reiser, Macromolecules 14, 95 (1981). 